Rapid Reviews COVID-19

\title{
Reviews of "Molecular Docking Studies of Aromatherapy Oils Against SARS-COV-2"
}

Kalirajan Rajagopal ${ }^{1}$, Umakanta Tripathy ${ }^{2}$, Janmejaya Rout ${ }^{3}$, Anand Kant Das ${ }^{4}$, Arun Sharma ${ }^{5}$

${ }^{1}$ Assistant Professor, JSS College of Pharmacy, Tamil Nadu, India, ${ }^{2}$ Indian Institute of Technology (Indian School of Mines): Indian Institute of Technology, Physics, India,

${ }^{3}$ Indian Institute of Technology (Indian School of Mines) Dhanbad, India,

${ }^{4}$ New York University, Abu Dhabi, ${ }^{5}$ Lyallpur Khalsa College, Biotechnology, India

Published on: Nov 22, 2020

DOI: $10.1162 / 2$ e3983f5.2a8dec63

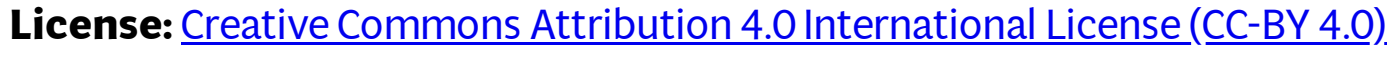


To read the original manuscript, click the link above.

Summary of Reviews: This study employs computational strategies to investigate whether aromatherapy-relevant compounds may be effective in suppressing SARS-CoV2 infection. While provocative, the data offered does not fully substantiate the claims made.

Reviewer 1 (Kalirajan Rajagopal) | प्र०

Reviewer 2 (Umakanta Tripathy, Janmejaya Rout, Anand Kant Das) | $\square \square \square \square \square$ Reviewer 3 (Arun Sharma) | 밈

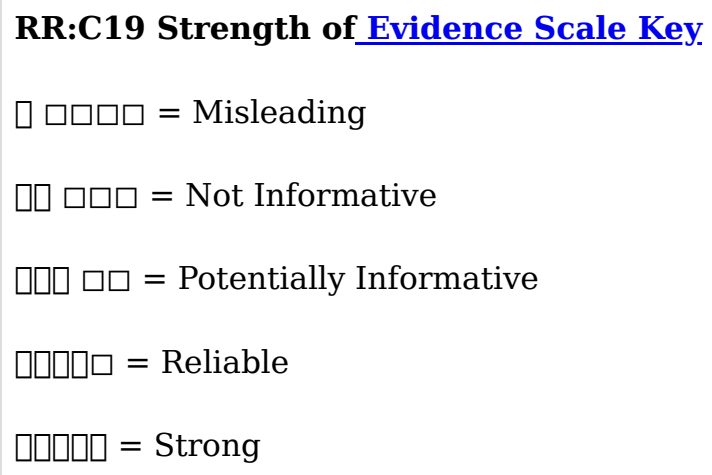

To read the reviews, click the links below. 